\title{
Tendencias de game learning analytics: una revisión sistemática de la literatura especializada
}

\section{Trends in game learning analytics: A systematic review of the specialized literature}

\section{Mayra Yadira Mejía Sierra*}

ALEXANDRO ESCUDERO-NAHÓN ${ }^{* *}$

\section{RicARDo CHAPARRo SÁNCHEZ ${ }^{* * *}$}

Este artículo presenta una revisión sistemática de la literatura especializada con el método de metasíntesis para conocer las tendencias teóricas y empíricas que pueden encontrarse en la literatura científica acerca de game learning analytics. La búsqueda se realizó en 17 bases de datos y se obtuvieron 153 resultados. Tras la aplicación de ciertos criterios de exclusión, se admitieron 17 artículos de investigación científica para el análisis. La información fue clasificada en tendencias de diseño, validación e implementación. Los hallazgos en diseño sugieren una tendencia a simular ambientes reales con el objetivo de validar, no solo el juego serio, sino también el aprendizaje obtenido aplicando mediciones pre- y postest. Se observó una implementación variada entre fines educativos, de capacitación o apoyo a personas con discapacidad. Asimismo, se emplearon juegos prediseñados y juegos de autor con interacciones individuales.

A systematic review of the specialized literature was carried out with the meta-synthesis method to know the theoretical and empirical trends that can be found in the scientific literature about game learning analytics. The search was carried out in 17 databases and 153 results were obtained. After applying certain exclusion criteria, 17 scientific research articles were admitted for analysis. The information was classified in design, validation, and implementation trends. The design findings suggest a tendency to simulate real environments to validate, not only serious play, but also the learning obtained by applying preand post-test measurements. A varied implementation was observed between educational purposes, training, or support for people with disabilities. Likewise, predesigned games and author's games with individual interactions were used.

\section{Palabras clave:}

game learning analytics, diseño, validación, implementación, revisión sistemática

\section{Keywords:}

game learning analytics, design, validation, implementation, systematic review

Recibido: 19 de mayo de 2021 | Aceptado para su publicación: 8 de febrero de 2022 |

Publicado: 12 de febrero de 2022

Cómo citar: Mejía Sierra, M. Y., Escudero-Nahón, A. y Chaparro Sánchez, R. (2022). Tendencias de game learning analytics: una revisión sistemática de la literatura especializada. Sinéctica, Revista Electrónica de Educación, (58), e1313. https://doi.org/10.31391/S2007-7033(2022)0058-006

\footnotetext{
*Estudiante del doctorado en Tecnología Educativa en la Universidad Autónoma de Querétaro, México. Líneas de investigación: game learning analytics. Correo electrónico: mmejia46@alumnos.uaq.mx/ https://orcid.org/0000-0003-0736-853X

** Doctor en Educación por la Universidad de Barcelona, España. Profesor-investigador en la Universidad Autónoma de Querétaro. Líneas de investigación: educación transdigital e intermodalidad educativa. Correo electrónico: alexandro. escudero@uaq.mx/http://orcid.org/0000-0001-8245-0838/https://www.transdigital.mx/aen/

*** Doctor en Innovación en Tecnología Educativa. Profesor en la Universidad Autónoma de Querétaro. Líneas de investigación: políticas, calidad y técnicas en tecnología educativa. Correo electrónico: rchapa@uaq.mx/ https://orcid. org/0000-0002-6842-2360
} 


\section{INTRODUCCIÓN}

T a primera aparición documentada del término game learning analytics se encontró en la Segunda Conferencia Internacional Games and Learning Alliance, que se llevó a cabo en París, Francia, en octubre de 2013. Esta conferencia fue organizada por la Serious Games Society junto con la Games and Learning Association y la Red de Excelencia en Serious Games, financiada por la Unión Europea (De Gloria \& Hutchison, 2013). Sin embargo, no fue sino hasta 2016 cuando la editorial Springer publicó el capítulo "Game learning analytics: Learning analytics for serious games", en el libro Learning, design, and technology (Freire et al., 2016).

Game learning analytics resulta de la unión de las técnicas serious game y learning analytics (Alonso-Fernández et al., 2019, 2020c). Actualmente, es un campo de investigación en crecimiento en el que intervienen tanto las ciencias computacionales como las sociales. Su popularidad ha crecido debido a que la conectividad a internet y el uso de dispositivos móviles han aumentado en casi todas las regiones del mundo. Además, los modelos educativos que incorporan esta técnica consideran que favorece el aprendizaje autónomo por vías lúdicas (Guzmán, Escudero-Nahón y Canchola-Magdaleno, 2020; Zainuddin et al., 2020).

Los serious game son un conjunto de actividades concebidas con fines educativos, explícitos, específicos y cuidadosamente pensados. No están destinados con propósitos de entretenimiento, diversión ni de manera informal. Sin embargo, esto no significa que no puedan ser entretenidos (Abt, 1987; Michael \& Chen, 2005). Su diseño debe contener objetivos pedagógicos y educativos claros, presentar una simulación que explique con claridad el dominio del juego, definir la progresión y los problemas que encontrará el jugador, exhibir una estética específica que lo haga atractivo al jugador, explicar las interacciones del jugador con la simulación del juego, así como especificar las condiciones de uso, el cómo, cuándo, dónde y quién utilizará el juego (Calvo-Morata et al., 2020).

Por su parte, el learning analytics o analítica del aprendizaje busca desarrollar y utilizar métodos de medición, recopilación, análisis y comunicación de datos, orientados a la comprensión y optimización del proceso de enseñanza-aprendizaje dentro de su entorno (Niemelä et al., 2020). Específicamente, en game learning analytics los datos obtenidos de la interacción del jugador con el juego serio pueden ser utilizados para evaluar a los estudiantes, predecir resultados de aprendizaje, validar los serious game y encontrar posibles mejoras en el diseño del juego (Alonso-Fernández et al., 2019).

Esta investigación documental presenta los resultados de una revisión sistemática aplicada en bases de datos científicos para conocer las tendencias teóricas y empíricas que pueden encontrarse en la literatura científica acerca de game learning analytics como estrategia educativa, desde su aparición, en 2015, hasta las tendencias actuales en su diseño y validación. Comprende las siguientes secciones: introducción al tema, descripción del método aplicado en la elaboración de la revisión sistemática (preguntas de investigación, término de búsqueda, fuentes de información, términos de exclusión e inclusión, análisis de duplicados y análisis de pertinencia para la selección de la literatura) y resultados, los cuales muestran las clasificaciones aplicadas en la extracción de la información proveniente de los artículos de investigación científicos seleccionados. Esta clasificación integró tendencias de diseño de juego serio y analítica del aprendizaje, 
tendencias de validación de juego serio y aprendizaje obtenido, y tendencias de implementación que consideran las características de los participantes en la investigación, área o intención de aprendizaje, uso de juegos de autor o prefabricados y la interacción del jugador con el juego serio individual o en parejas. Finalmente, en las discusiones exponemos la utilidad de los resultados, así como líneas de investigación futuras.

\section{MÉTODO}

La revisión sistemática de la literatura especializada se elaboró con base en la metodología de metasíntesis, la cual integra los resultados de estudios cualitativos y procesa los datos obtenidos de forma inductiva con la finalidad de interpretar hallazgos relevantes (Leary \& Walker, 2018). El principal objetivo fue comprender las tendencias de diseño, validación e implementación de game learning analytics desde 2013, año en el que se registran las primeras investigaciones referentes al tema, hasta 2020. El procedimiento incluyó cuatro etapas y seis pasos, los cuales se detallan en la figura 1 (Grijalva et al., 2019).

Etapas de la revisión sistemática

\begin{tabular}{|c|c|c|c|}
\hline Planeación & Selección & Extracción & Resultac \\
\hline $\begin{array}{l}\text { Paso 1. Plantear } \\
\text { las preguntas de } \\
\text { investigación }\end{array}$ & $\begin{array}{l}\text { Paso } 3 . \\
\text { Elegir las fuentes } \\
\text { de información }\end{array}$ & $\begin{array}{l}\text { Paso } 5 . \\
\text { Concentrar y } \\
\text { analizar los datos }\end{array}$ & $\begin{array}{l}\text { Paso } 6 . \\
\text { Determinar } \\
\text { hallazgos }\end{array}$ \\
\hline $\begin{array}{l}\text { Paso } 2 \text {. } \\
\text { Definir el término } \\
\text { de búsqueda }\end{array}$ & $\begin{array}{l}\text { Paso } 4 . \\
\text { Seleccionar la } \\
\text { literatura } \\
\text { especializada }\end{array}$ & & \\
\hline
\end{tabular}

Pasos de la revisión sistemática

Figura 1. Etapas y pasos de la revisión sistemática.

\section{Planeación}

Plantear las preguntas de investigación

La pregunta de investigación que nos guio fue: ¿cuáles son las tendencias de diseño, validación e implementación de game learning analytics de 2015 a 2020 ?

\section{Definir el término de búsqueda}

El término empleado en la búsqueda de la literatura especializada en las bases de datos científicas utilizó el método booleano. La cadena de búsqueda ingresada en las bases de datos científicas fue "game learning analytics". Utilizamos las comillas para obtener una coincidencia exacta de la frase. En caso de no utilizar comillas, los resultados de la búsqueda mostraban publicaciones que contenían entre sus términos de búsqueda las palabras game, learning o analytics en algún lugar del texto, lo que volvía a los resultados poco relevantes para nuestros objetivos. Seleccionamos los textos científicos que presentaban el término de búsqueda exacto ("game learning analytics") en el título, el resumen o el contenido de la literatura especializada. 


\section{Selección}

Fuentes de información

Como fuentes de información, seleccionamos 17 bases de datos e incluimos algunas de las principales para educación, informática e investigación científica general: Scopus, ScienceDirect, SpringerLink, Web of Science, Conricyt, Redalyc, Directory of Open Access Journals, IEEE Xplore, Dialnet, Emerald Insight, EBSCOhost, SciELO, Latindex, Association for Computing Machinery, Education Resources Information Center, Cambridge Journals Online y Oxford University Press.

Selección de la literatura especializada

$\mathrm{Al}$ ingresar en las citadas bases de datos, la consulta de búsqueda "game learning analytics" obtuvo 153 resultados, a los cuales les aplicamos los siguientes criterios de exclusión e inclusión, análisis de duplicados y análisis de pertinencia.

Criterios de exclusión e inclusión

Las características para incluir literatura en la revisión sistemática fueron: artículos de investigación científica en inglés y español. Por otro lado, para determinar qué artículos de investigación científica no serían incluidos, consideramos la pertinencia, es decir, los artículos que, aunque cumplían los criterios de inclusión, no eran relevantes para la investigación debido a su escasa información sobre el diseño, la validación y la implementación de game learning analytics.

Para la verificación y eliminación de duplicados, nos apoyamos en la herramienta check for duplicates del gestor de referencias bibliográficas Mendeley. La revisión del título y el resumen manualmente para garantizar una minuciosa eliminación de duplicados en la siguiente matriz de duplicados nos permitió conocer cuántos, cuáles y en qué bases de datos se encuentran los artículos duplicados. Los resultados alcanzados al aplicar los criterios descritos se incluyen en la figura 2.

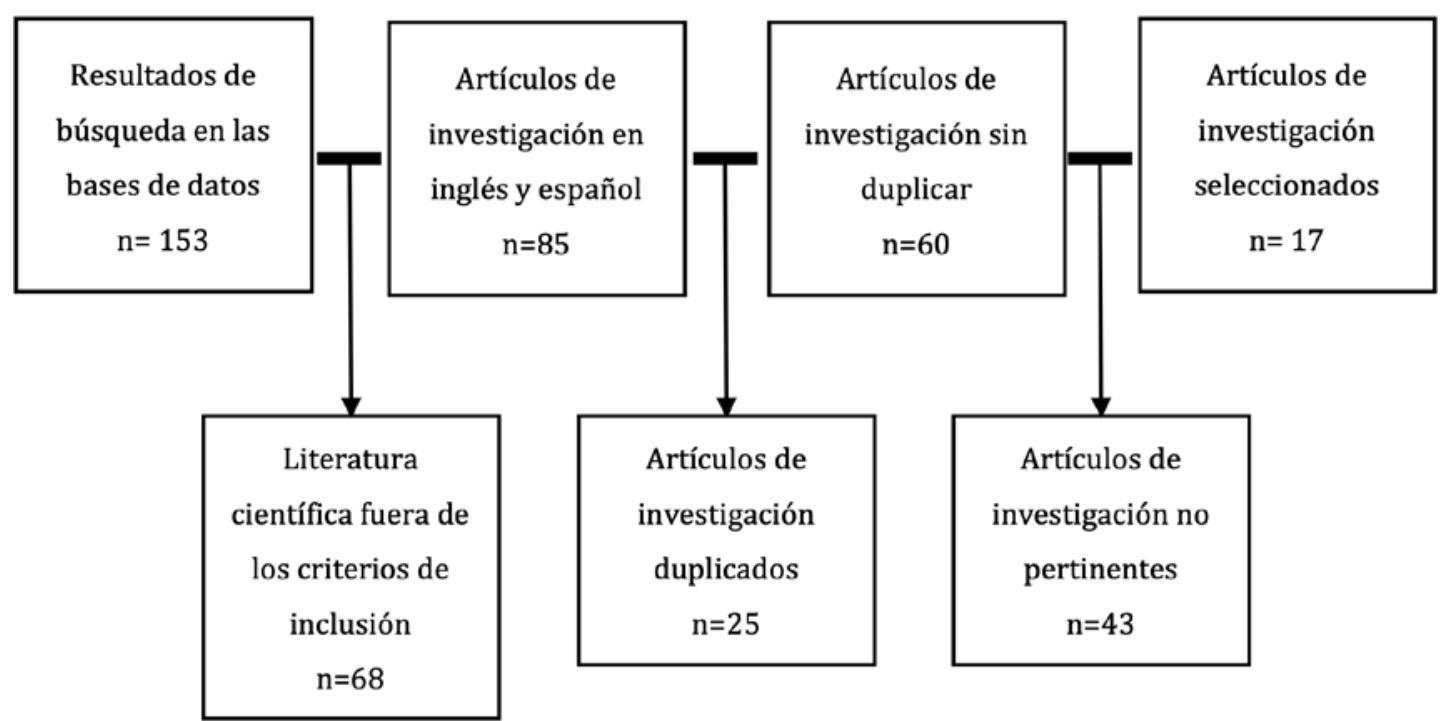

Figura 2. Proceso de selección de literatura científica para la revisión sistemática. 
Análisis de riesgo de sesgo

Inspirados en Sterne y colaboradores (2016), realizamos un análisis de riesgo de sesgo. En la tabla 1 se definen los dominios y la descripción de los posibles sesgos de la investigación: información complementaria, término de búsqueda, diseño, validación e implementación.

Tabla 1. Descripción de dominios de posibles sesgos

\begin{tabular}{|c|c|}
\hline $\begin{array}{c}\text { Dominio } \\
\text { Información } \\
\text { complementaria }\end{array}$ & $\begin{array}{c}\text { Descripción } \\
\text { Cuando un artículo de investigación resul- } \\
\text { tado de las búsquedas en las bases de datos } \\
\text { científicas se excluye por contener información } \\
\text { adicional a la definida en los objetivos de la } \\
\text { revisión sistemática }\end{array}$ \\
\hline $\begin{array}{c}\text { Término de } \\
\text { búsqueda }\end{array}$ & $\begin{array}{c}\text { Cuando un artículo de investigación resultado } \\
\text { de las búsquedas en las bases de datos cientí- } \\
\text { ficas se excluye por contener pocas menciones } \\
\text { del término game learning analytics }\end{array}$ \\
\hline Diseño & $\begin{array}{c}\text { Cuando un artículo de investigación científica } \\
\text { resultado de las búsquedas en las bases de } \\
\text { datos científicas se excluye por no detallar } \\
\text { aspectos de codificación del serious game } \\
\text { utilizado }\end{array}$ \\
\hline Validación & $\begin{array}{c}\text { Cuando un artículo de investigación científica } \\
\text { resultado de las búsquedas en las bases de } \\
\text { datos científicas se excluye por no detallar } \\
\text { algoritmos implementados en el proceso de } \\
\text { learning analytics }\end{array}$ \\
\hline Implementación & $\begin{array}{c}\text { Cuando un artículo de investigación científica } \\
\text { resultado de las búsquedas en las bases de } \\
\text { datos científicas se excluye por no detallar el } \\
\text { área de implementación }\end{array}$ \\
\hline
\end{tabular}

\section{Extracción}

\section{Concentración y análisis de datos}

Los artículos de investigación científica seleccionados fueron descargados e identificados integrando el nombre o abreviación de la base de datos de procedencia, un número identificador seguido de un guion y del número de publicaciones totales encontradas (ejemplo: Scopus 1-11).

La información esencial de los artículos de investigación científica se concentró en una matriz con los siguientes aspectos generales: ID, nombre, autor (es), palabras clave, fecha de publicación, doi, revista, número de indexación, introducción, método, resultados, conclusiones, así como los siguientes aspectos propios de la pregunta de investigación: diseño, validación e implementación, además de consideraciones para herramientas o software y otros comentarios.

De cada uno de los artículos de investigación científica seleccionados, recopilamos los datos y categorizamos los resultados en función de las preguntas de investigación con base en los siguientes criterios:

-Diseño de game learning analytics

-Validación de game learning analytics

-Implementación de game learning analytics 


\section{RESULTADOS}

La búsqueda referente al término game learning analytics se realizó en 17 bases de datos, de las cuales obtuvimos 153 resultados. Estos fueron filtrados empleando criterios de exclusión e inclusión, análisis de duplicados y pertinencia, lo que arrojó un total de 17 artículos de investigación científica. La tabla 2 contiene los hallazgos por base de datos, así como los artículos

Tabla 2. Artículos encontrados e incluidos por base de datos

\begin{tabular}{|c|c|c|}
\hline Bases de datos & $\begin{array}{c}\text { Literatura } \\
\text { encontrada }\end{array}$ & $\begin{array}{c}\text { Literatura } \\
\text { incluida }\end{array}$ \\
\hline Scopus & 28 & 9 \\
\hline Science Direct & 5 & 2 \\
\hline Springer & 26 & 1 \\
\hline Web of Science & 11 & 0 \\
\hline Conricyt & 44 & 1 \\
\hline Redalyc & 0 & 0 \\
\hline DOAJ & 1 & 1 \\
\hline IEEE & 11 & 0 \\
\hline Dialnet & 5 & 0 \\
\hline Emerald & 1 & 0 \\
\hline EBSCO & 7 & 0 \\
\hline SciELO & 0 & 0 \\
\hline Latindex & 0 & 0 \\
\hline ACM & 5 & 3 \\
\hline ERIC & 9 & 0 \\
\hline CJO & 0 & 0 \\
\hline OUP & 0 & 0 \\
\hline Total & 153 & 17 \\
\hline
\end{tabular}

Gráfica. Análisis de duplicados de la literatura pertinente

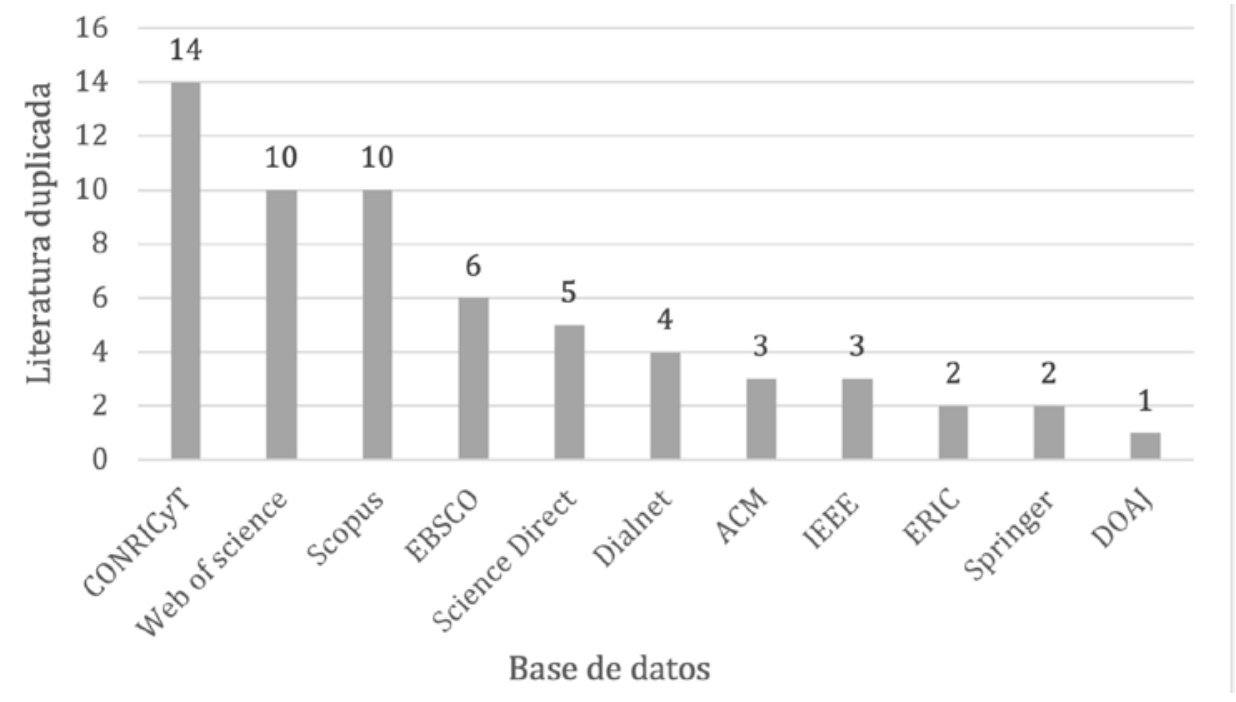


El análisis de duplicados fue realizado considerando las bases de datos que arrojaron algún resultado al término de búsqueda ingresado. Las bases de datos que no presentaron resultados de búsqueda fueron: Redalyc, SciELO, Latindex, Cambridge Journals Online, Oxford University Press y Emerald. Como observamos, la base datos con mayor cantidad de artículos duplicados es Conricyt.

Tendencias de diseño

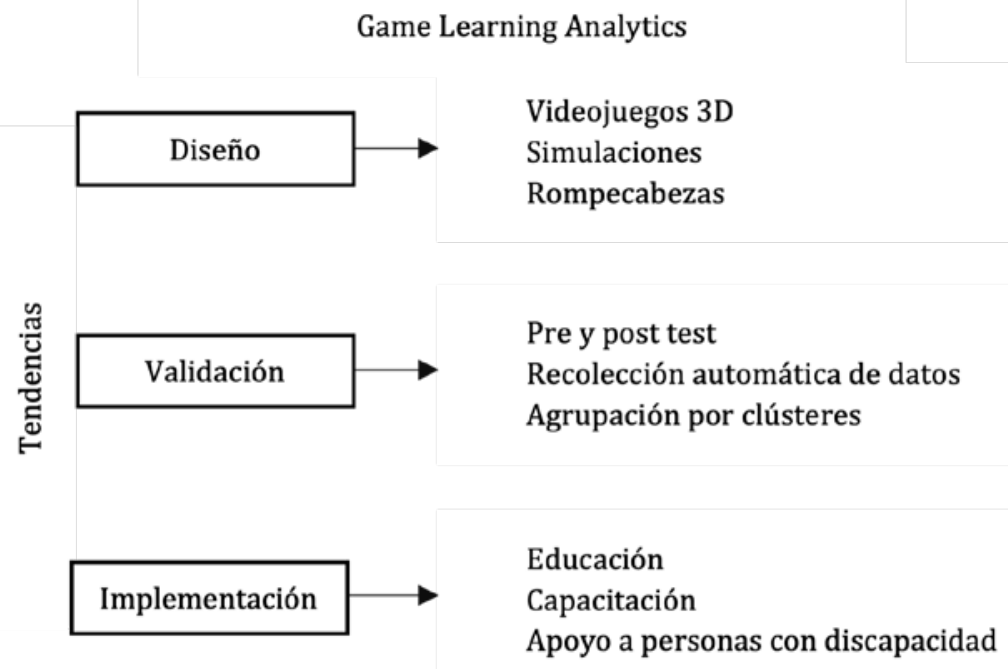

Figura 3. Dimensiones del estudio.

Los hallazgos de esta revisión sistemática indicaron que hay tres categorías de análisis principales: diseño, validación e implementación; esas categorías contienen subcategorías (ver figura 3). Las tendencias de diseño de los serious games empleados en game learning analytics se centraron en el desarrollo de videojuegos 3D, simulaciones, rompecabezas y juegos de conexión. La validación de los game learning analytics analizados en nuestra investigación fue llevada a cabo mediante pre- y postest, recolección automática de datos, agrupación por clústeres y minería de datos. Los game learning analytics fueron utilizados para la educación, capacitación y apoyo a personas con discapacidad intelectual.

Toda implementación de game learning analytics requiere un serious game. Estos no siempre son diseñados específicamente con learning analytics. Las investigaciones analizadas mostraron una tendencia a los videojuegos 3D, simulaciones, rompecabezas y juegos de conexión; todos ellos son juegos prefabricados con una aplicación externa de learning analytics. En la tabla 3 se detallan el autor, juego, tipo, así como base pedagógica desde la cual fue diseñado. 
Tabla 3. Diseño de los juegos empleados en game learning analytics

\begin{tabular}{|c|c|c|c|}
\hline Autor y año & Nombre & Tipo & Base pedagógica \\
\hline Alonso-Fernández et al., 2019 & DownTown & Videojuego 3D & $\begin{array}{l}\text { Características y barreras } \\
\text { cognitivas más comunes de los } \\
\text { usuarios }\end{array}$ \\
\hline Alonso-Fernández, 2020a & Conectados & $\begin{array}{l}\text { Rompecabezas } \\
\text { 3D }\end{array}$ & $\begin{array}{c}\text { Literatura referente a las } \\
\text { situaciones, escenarios y roles } \\
\text { comunes del acoso, así como } \\
\text { del ciberacoso }\end{array}$ \\
\hline Calvo-Morata et al., 2019 & First Aid Game & Simulación & $\begin{array}{l}\text { Pautas definidas por el Euro- } \\
\text { pean Resuscitation Council }\end{array}$ \\
\hline Capatina et al., 2018 & Simbound & Simulación & $\begin{array}{l}\text { Modelo de aprendizaje colabo- } \\
\text { rativo }\end{array}$ \\
\hline Cloude et al., 2020 & Crystal Island & Video juego 3D & $\begin{array}{c}\text { Estándares esenciales del curso } \\
\text { de estudio estándar para mi- } \\
\text { crobiología de octavo grado }\end{array}$ \\
\hline Niemelä et al., 2020 & GraphoLearn & Conexiones & Normas del idioma finés \\
\hline Peddycord-Liu et al., 2017 & ST Math & Rimpecabezas & Plan de estudio \\
\hline Rotaru et al., 2018 & COSMOS & Video juego 3D & $\begin{array}{c}\text { Pruebas neuropsicológicas: } 15 \\
\text { Objects Test }\end{array}$ \\
\hline Ruipérez \& Kim, 2020 & Shadowspect & Videojuego 3D & Plan de estudio \\
\hline
\end{tabular}

\section{Tendencias de validación}

Entre los métodos identificados para validar el serious game y el aprendizaje obtenido por el jugador al interactuar con este, destaca la aplicación de experimentos previos y posteriores. Los experimentos consistieron en un cuestionario previo (pretest), seguido de la interacción del jugador con el serious game para, finalmente, volver a aplicar el cuestionario inicial (postest). Los cuestionarios fueron validados de modo previo. En caso de que se careciera de un instrumento de validación para el cuestionario, este se elaboró ad-hoc (Alonso-Fernández et al., 2020; Alonso-Fernández et al., 2020c; Calvo-Morata et al., 2019; Melo et al., 2019; Rotaru et al., 2018).

La aplicación de pre- y postest es una actividad que consume recursos, ya que, en general, se aplican en un entorno externo al serious game y hacen necesaria la captura y el procesamiento de datos con herramientas externas, lo que origina la necesidad de integrar pre- y postest en el entorno del serious game o buscar alternativas para su optimización (Alonso-Fernández et al., 2020a).

Otro método identificado para validar el aprendizaje consistió en la recolección de los datos de interacción del jugador con el serious game mediante la técnica de seguimiento de game analytics, comúnmente usada en juegos comerciales con fines de rentabilidad, aunque puede adaptarse a fines educativos. La información obtenida de los datos de interacción fue utilizada tanto para evaluar y mejorar el juego como para dar seguimiento al progreso de los estudiantes e incluso evaluarlo (Alonso-Fernández et al., 2020c; Tsikinas \& Xinogalos, 2020). 
En determinadas situaciones, la recolección de los datos de interacción demostró ser indispensable cuando el aprendizaje obtenido de la interacción del jugador con el juego serio no pudo medirse con pre- y postest (Alonso-Fernández et al., 2019). Si bien es cierto que el uso de pre- y postest es una herramienta efectiva para la recolección de datos, la aplicación de xAPI-SG representa una alternativa para recolectar datos, ya que recurre a estadísticas agregadas de alto nivel sobre el rendimiento del jugador y el equipo (Alonso-Fernández et al., 2019, 2020a, 2020b; Calvo-Morata et al., 2019; Cano, Fernández-Manjón \& García-Tejedor, 2018; Charleer et al., 2018).

Entre la variedad de alternativas disponibles para validar el aprendizaje, encontramos el método de agrupación en clústeres, por lo regular utilizado en la analítica del aprendizaje. Esto consistió en el agrupamiento jerárquico para clasificar estudiantes, su aprendizaje, progreso en el juego y el proceso de aprendizaje de los jugadores. Se empleó una estructura tipo árbol y no se aconsejó para conjuntos de datos de gran tamaño, ya que arrojó figuras arbitrarias. Por otro lado, se aplicó el método de minería de datos K-medias, en el cual cada observación pertenece a un grupo de acuerdo con la media más cercana y presenta agrupación en una capa con subconjuntos geométricamente cerrados de fácil interpretación (Niemelä et al., 2020).

Además de los métodos mencionados, fue posible validar el aprendizaje resultado de la interacción del jugador con el juego serio al aplicar cuestionarios de opción múltiple diseñados para medir el aprendizaje esperado, en los que, al obtener la respuesta, se mostraron videos adicionales con los procedimientos correctos (AlonsoFernández et al., 2019).

La minería de datos también fue empleada para validar el aprendizaje obtenido por el jugador al finalizar la interacción con el juego serio: modelos de clasificación, como son árboles de decisión, regresión logística y clasificador Naïve Bayes para determinar aprobación-reprobación, y modelos lineales, como lo son árboles de regresión, regresión lineal y regresión de vectores de soporte con núcleos no lineales para conocer el puntaje exacto (Alonso-Fernández et al., 2019, 2020b).

\section{Tendencias de implementación}

En esta categoría se concentraron las tendencias de implementación para game learning analytics. La tabla 4 muestra el autor, año de la investigación, una breve descripción de los participantes, el propósito y el tiempo de implementación. Durante la aplicación del game learning analytics, usamos serious game, cuyo diseño no fue enfocado específicamente a las investigaciones documentadas, es decir, utilizamos serious game prefabricados (Alonso-Fernández et al., 2020a, 2020c; Niemelä et al., 2020; Ruipérez-Valiente \& Kim, 2020). 
Tabla 4. Implementación de game learning analytics

\begin{tabular}{|c|c|c|c|}
\hline Año y autor & $\begin{array}{l}\text { Participante del } \\
\text { estudio }\end{array}$ & Propósito de la implementación & $\begin{array}{l}\text { Tiempo de } \\
\text { implementa- } \\
\text { ción }\end{array}$ \\
\hline Alonso-Fernández et al., 2019 & $\begin{array}{l}\text { Adultos entre } 18 \text { y } \\
45 \text { años con discapa- } \\
\text { cidad intelectual }\end{array}$ & $\begin{array}{l}\text { Orientar en el uso del sistema de } \\
\text { transporte público }\end{array}$ & 3 horas \\
\hline Alonso-Fernández, 2020a & $\begin{array}{l}\text { Estudiantes de K-12 } \\
\text { (equivalente a pri- } \\
\text { maria y secundaria) }\end{array}$ & $\begin{array}{l}\text { Aumentar la conciencia sobre el } \\
\text { acoso escolar }\end{array}$ & 55 minutos \\
\hline Calvo-Morata et al., 2019 & $\begin{array}{l}\text { Estudiantes entre } 12 \\
\text { y } 17 \text { años }\end{array}$ & $\begin{array}{l}\text { Instruir en maniobras de reani- } \\
\text { mación cardiopulmonar }\end{array}$ & 30 minutos \\
\hline Capatina et al., 2018 & Empleados & $\begin{array}{l}\text { Mejorar las habilidades colabo- } \\
\text { rativas }\end{array}$ & No especifica \\
\hline Cloude et al., 2020 & $\begin{array}{l}\text { Estudiantes univer- } \\
\text { sitarios }\end{array}$ & $\begin{array}{l}\text { Mejorar la transferencia y reten- } \\
\text { ción de habilidades de razona- } \\
\text { miento científico }\end{array}$ & 90 minutos \\
\hline Niemelä et al., 2020 & $\begin{array}{l}\text { Estudiantes de } 6 \text { a } \\
9 \text { años }\end{array}$ & $\begin{array}{l}\text { Entrenar las conexiones entre } \\
\text { los sonidos del habla y las letras }\end{array}$ & 10 minutos \\
\hline Peddycord-Liu et al., 2017 & $\begin{array}{l}\text { Estudiantes de tercer } \\
\text { grado }\end{array}$ & $\begin{array}{c}\text { Apoyar en la enseñanza del plan } \\
\text { de estudios de matemáticas }\end{array}$ & 60 minutos \\
\hline Rotaru et al., 2018 & $\begin{array}{l}\text { Adultos mayores de } \\
60 \text { años cognitiva- } \\
\text { mente sanos }\end{array}$ & $\begin{array}{l}\text { Pruebas neuropsicológicas com- } \\
\text { putarizadas }\end{array}$ & 25 minutos \\
\hline Ruipérez \& Kim, 2020 & $\begin{array}{l}\text { Estudiantes de pri- } \\
\text { mer año de bachi- } \\
\text { llerato }\end{array}$ & $\begin{array}{l}\text { Desarrollar habilidades de razo- } \\
\text { namiento geométrico, dimensio- } \\
\text { nal y espacial }\end{array}$ & 75 minutos \\
\hline
\end{tabular}

Además de las aplicaciones empíricas de game learning analytics, se reportó la elaboración de un marco de trabajo para diseñar game learning analytics para personas con discapacidad desde un enfoque teórico centrado en fomentar la elaboración de serious game en un entorno de aprendizaje inclusivo diseñado con prudencia, competencias adaptables y mensurables para satisfacer las necesidades de los jugadores (Nguyen, Gardner \& Sheridan, 2018).

Respecto a la interacción de los estudiantes con el juego, esta sucedió de forma individual (Alonso-Fernández et al., 2019, 2020a, 2020b; Calvo-Morata et al., 2019, 2020; Cano, Fernández-Manjón \& García-Tejedor, 2018; Cloude et al., 2020; Niemelä et al., 2020; Peddycord-Liu et al., 2017; Rotaru et al., 2018) o colaborativa. Los resultados son diferentes, no necesariamente superiores unos frente a otros; mientras que la interacción individual permitió un mayor avance en la realización de los juegos y en la participación, la interacción en binas demostró mayor exploración del juego (Ruipérez-Valiente \& Kim, 2020).

Como resultado del análisis de los artículos de investigación científica seleccionados para la revisión sistemática, elaboramos la figura 4, en la cual establecemos la mecánica y el proceso a seguir en la elaboración de game learning analytics. 


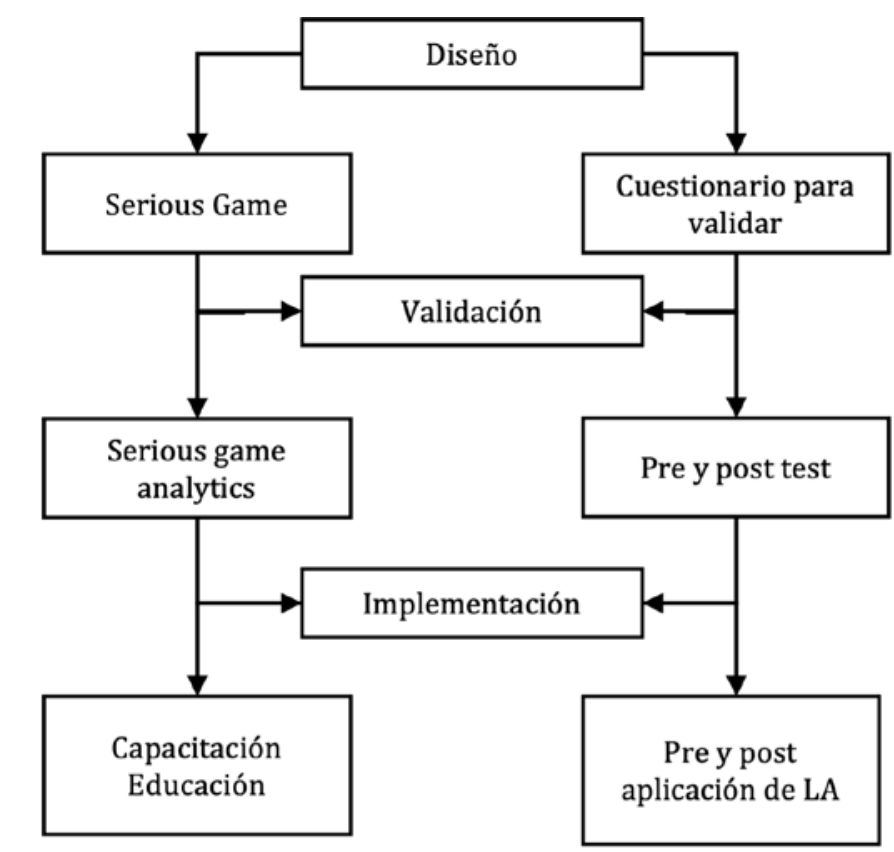

Figura 4. Tendencias de diseño, validación e implementación de game learning analytics.

\section{DiSCUSIÓN Y CONCLUSIONES}

Esta revisión sistemática de la literatura especializada con el método de metasíntesis tuvo el objetivo de conocer las tendencias teóricas y empíricas vigentes en la literatura científica acerca de game learning analytics. Destacó el hecho de que el diseño preponderante fueron videojuegos 3D y simulaciones. Esto coincide con la literatura especializada respecto a una clara incorporación de tecnología digital inmersiva en los procesos de aprendizaje, y cuyo estadio más sofisticado es el recientemente popular metaverso (Barry et al., 2015; Kanematsu, 2014; Kuksa \& Childs, 2014). Sin embargo, al revisar el año de publicación de esa literatura, fue evidente que la tendencia inmersiva, por lo menos en educación, data ya de cerca de una década, lo que puede interpretarse como una tendencia poco novedosa, pero inconclusa.

En cuanto a la validación de game learning analytics, fue evidente que hay una marcada tendencia a usar procedimientos e instrumentos cuantitativos. Lo anterior coincide con las propuestas de análisis del big data, pero es contrario al discurso educativo centrado en el aprendizaje. En otras palabras, en la validación se ha puesto el acento en la optimización del artefacto, pero no en conocer con amplitud y profundidad qué problemas tiene el alumnado al experimentar un game learning analytics y cómo resuelve esos problemas. Es necesario introducir propuestas de validación del artefacto con enfoque cualitativo, así como iniciar estudios cualitativos sobre el aprendizaje con este tipo de modelos (Lew \& Saville, 2021; Xu et al., 2021).

El tercer hallazgo radica en las áreas en las que se han implementado estos prototipos. Llama la atención que los ámbitos de educación formal y no formal sean igualmente abordados. Esto es sintomático de un desplazamiento de interés hacia métodos alternativos de aprendizaje, no solo de prototipos, sino de contextos educativos. Los tres hallazgos anteriores, en general, coinciden con las tendencias que la literatura especializada en tecnología educativa está desarrollando: nuevos modelos 
y prototipos educativos aplicados en contextos formales, no formales e informales. Sin embargo, una sugerencia relevante es que se realicen más estudios cualitativos (Hallinger et al., 2020; Kaivo-oja, 2017).

En conclusión, en este trabajo explicamos las tendencias de diseño, validación e implementación de game learning analytics. El área de investigación para este término es reciente y está en crecimiento. Los artículos de investigación científica que desarrollan de manera amplia el tema son escasos y se encuentran considerablemente duplicados en las bases de datos.

Comprender las tendencias de diseño, validación e implementación de game learning analytics favorece la consolidación de término, y sirve como guía no solo para investigadores de áreas educativas, sino también para desarrolladores o investigadores interesados en elaborar este tipo de recursos. Es importante destacar que las tendencias de diseño para esta analítica de aprendizaje se inclinan a la selección e implementación de un juego serio más que a la elaboración de este, lo cual facilita y pone al alcance el uso de game learning analytics con fines educativos para docentes que cuentan con poca capacitación tecnológica en la elaboración de serious game y learning analytics.

El encontrar pocos autores y artículos de investigación científica dificultó la elaboración de la revisión sistemática. Sin embargo, abre la puerta a trabajos futuros, en especial en lo referente a la base pedagógica sobre la cual se desarrollará game learning analytics. Si bien algunas investigaciones basan la implementación y validación en estándares, normas idiomáticas y literatura diversa, ninguna menciona la aplicación de un modelo educativo como tal. Podemos concluir que las investigaciones documentadas y analizadas en esta revisión sistemática se inclinan hacia una aplicación empírica. Sin duda, es necesario ampliar tanto la investigación como su publicación.

\section{REFERENCIAS BIBLIOGRÁFICAS}

Abt, C. C. (1987). Serious games. EUA: University Press of America.

Alonso-Fernández, C., Calvo-Morata, A., Freire, M., Martínez-Ortiz, I. \& Fernández-Manjón, B. (2020a). Evidence-based evaluation of a serious game to increase bullying awareness. Interactive Learning Environments, vol. 28, núm. 1, pp. 1-11. https://doi.org/10.1080/10494820.2020.1799031

Alonso-Fernández, C., Martínez-Ortiz, I., Caballero, R., Freire, M. \& FernándezManjón, B. (2020b). Predicting students' knowledge after playing a serious game based on learning analytics data: A case study. Journal of Computer Assisted Learning, vol. 36, núm. 3, pp. 350-358. https://doi.org/10.1111/ jcal.12405

Alonso-Fernández, C., Pérez-Colado, I. J., Calvo-Morata, A., Freire, M., Ortiz, I. M. \& Manjón, B. F. (2020c). Applications of Simva to simplify serious games validation and deployment. Revista Iberoamericana de Tecnologías del Aprendizaje, vol. 15, núm. 3, pp. 161-170. https://doi.org/10.1109/RITA.2020.3008117

Alonso-Fernández, C., Cano, A. R., Calvo-Morata, A., Freire, M., Martínez-Ortiz, I. \& Fernández-Manjón, B. (2019). Lessons learned applying learning analytics to assess serious games. Computers in Human Behavior, vol. 99, núm. 9, pp. 301-309. https://doi.org/10.1016/j.chb.2019.05.036 
Barry, D. M., Ogawa, N., Dharmawansa, A., Kanematsu, H., Fukumura, Y., Shirai, T., Yajima, K. \& Kobayashi, T. (2015). Evaluation for students' learning manner using eye blinking system in Metaverse. Procedia Computer Science, vol. 60, pp. 1195-1204. https://doi.org/10.1016/j.procs.2015.08.181

Calvo-Morata, A., Alonso-Fernández, C., Freire-Morán, M., Martínez-Ortiz, I. \& Fernández-Manjón, B. (2019). Game learning analytics, facilitating the use of serious games in the class. Revista Iberoamericana de Tecnologías del Aprendizaje, vol. 14, núm. 4, pp. 168-176. https://doi.org/10.1109/ RITA.2019.2952296

Calvo-Morata, A., Rotaru, D. C., Alonso-Fernández, C., Freire-Morán, M., Martínez-Ortiz, I., Fernández-Manjón, B., Alonso-Fernandez, C., Freire-Moran, M., Martinez-Ortiz, I. \& Fernandez-Manjon, B. (2020). Validation of a cyberbullying serious game using game analytics. IEEE Transactions on Learning Technologies, vol. 13, núm. 1, pp. 186-197. https://doi.org/10.1109/ TLT.2018.2879354

Cano, A. R., Fernández-Manjón, B. \& García-Tejedor, Á. J. (2018). Using game learning analytics for validating the design of a learning game for adults with intellectual disabilities. British Journal of Educational Technology, vol. 49, núm. 4, pp. 659-672. https://doi.org/10.1111/bjet.12632

Capatina, A., Bleoju, G., Rancati, E. \& Hoareau, E. (2018). Tracking precursors of learning analytics over serious game team performance ranking. Behaviour \& Information Technology, vol. 37, núm. 10-11, pp. 1008-1020. https://doi.org/ 10.1080/0144929X.2018.1474949

Charleer, S., Verbert, K., Gutiérrez, F. \& Gerling, K. (2018). Towards an open standard for gameplay metrics. CHI PLAY 2018-Proceedings of the 2018 Annual Symposium on Computer-Human Interaction in Play Companion Extended Abstracts (pp. 399-406). https://doi.org/10.1145/3270316.3271529

Cloude, E. B., Dever, D. A., Wiedbusch, M. D. \& Azevedo, R. (2020). Quantifying scientific thinking using multichannel data with crystal island: Implications for individualized game-learning analytics. Frontiers in Education, vol. 5 (november), pp. 1-21. https://doi.org/10.3389/feduc.2020.572546

De Gloria, A. \& Hutchison, D. (2013). Games and learning alliance (A. De Gloria [ed.], vol. 41, núm. 1). https://doi.org/10.1007/978-3-319-12157-4

Freire, M., Serrano-Laguna, Á., Iglesias, B. M., Martínez-Ortiz, I., Moreno-Ger, P. \& Fernández-Manjón, B. (2016). Game learning analytics: Learning analytics for serious games in learning, design, and technology. En M. J. Spector, B. B. Lockee \& M. D. Childress [eds.]. Learning, design, and technology. Springer, Cham. https://doi.org/10.1007/978-3-319-17727-4

Grijalva, P. K., Cornejo, G. E., Gómez, R., Real, K. P., Fernández, A., Gomez, R. R., Real, K. P. \& Fernandez, A. (2019). Herramientas colaborativas para revisiones sistemáticas. Revista Espacios, vol. 40, núm. 45, p. 9. https://www.revistaespacios.com/a19v40n25/a19v40n25p09.pdf

Guzmán, M. A., Escudero-Nahón, A. y Canchola-Magdaleno, S. L. (2020). “Gamificación" de la enseñanza para ciencia, tecnología, ingeniería y matemáticas: cartografía conceptual. Sinéctica, Revista Electrónica de Educación, núm. 54, pp. 1-20. https://doi.org/10.31391/S2007-7033(2020)0054-002

Hallinger, P., Wang, R., Chatpinyakoop, C., Nguyen, V.-T. \& Nguyen, U.-P. (2020). A bibliometric review of research on simulations and serious games used in educating for sustainability, 1997-2019. Journal of Cleaner Production, vol. 256, 120358. https://doi.org/10.1016/j.jclepro.2020.120358 
Kaivo-oja, J. (2017). Towards better participatory processes in technology foresight: How to link participatory foresight research to the methodological machinery of qualitative research and phenomenology? Futures, vol. 86, pp. 94-106. https://doi.org/10.1016/j.futures.2016.07.004

Kanematsu, H., Kobayashi, T., Barry, D. M., Fukumura, Y., Dharmawansa, A. \& Ogawa, N. (2014). Virtual STEM Class for nuclear safety education in Metaverse. Procedia Computer Science, vol. 35, pp. 1255-1261. https://doi. org/10.1016/j.procs.2014.08.224

Kuksa, I. \& Childs, M. (2014). 8-Virtual worlds in education. En I. Kuksa \& M. Childs (eds.). Making Sense of Space (pp. 101-118). Chandos Publishing. https://doi.org/10.1533/9781780634067.3.101

Leary, H. \& Walker, A. (2018). Meta-analysis and meta-synthesis methodologies: Rigorously piecing together research. TechTrends, vol. 62, núm. 5, pp. 525-534. https://doi.org/10.1007/s11528-018-0312-7

Lew, C. \& Saville, A. (2021). Game-based learning: Teaching principles of economics and investment finance through Monopoly. The International Journal of Management Education, vol. 19, núm. 3, 100567. https://doi.org/10.1016/j. ijme.2021.100567

Melo, S. A., Paes, A., Clua, E. W. G., Kohwalter, T. C. \& Murta, L. G. P. (2019). Detecting long-range cause-effect relationships in game provenance graphs with graph-based representation learning. Entertainment Computing, vol. 32 (enero), 100318. https://doi.org/10.1016/j.entcom.2019.100318

Michael, D. \& Chen, S. (2005). Serious games: Games that educate, train, and inform. Muska \& Lipman/Premier-Trade. https://doi.org/10.5555/1051239

Nguyen, A., Gardner, L. A. \& Sheridan, D. (2018). A framework for applying learning analytics in serious games for people with intellectual disabilities. British Journal of Educational Technology, vol. 49, núm. 4, pp. 673-689. https:// doi.org/10.1111/bjet.12625

Niemelä, M., Kärkkäinen, T., Äyrämö, S., Ronimus, M., Richardson, U. \& Lyytinen, H. (2020). Game learning analytics for understanding reading skills in transparent writing system. British Journal of Educational Technology, vol. 51, núm. 6, pp. 2376-2390. https://doi.org/10.1111/bjet.12916

Peddycord-Liu, Z., Cody, C., Kessler, S., Barnes, T., Lynch, C. F. \& Rutherford, T. (2017). Using serious game analytics to inform digital curricular sequencing: What math objective should students play next? Proceedings of the Annual Symposium on Computer-Human Interaction in Play (pp. 195-204). https:// doi.org/10.1145/3116595.3116620

Rotaru, D. C., García-Herranz, S., Morán, M. F., Martínez-Ortiz, I., Fernández-Manjón, B. \& Carmen Díaz, M. (2018). Using game technology to automatize neuropsychological tests and research in active aging. ACM International Conference Proceeding Series (pp. 65-70). https://doi.org/10.1145/3284869.3284887

Ruipérez-Valiente, J. A. \& Kim, Y. J. (2020). Effects of solo vs. collaborative play in a digital learning game on geometry: Results from a K12 experiment. Computers and Education, vol. 159 (octubre), 104008. https://doi.org/10.1016/j. compedu.2020.104008

Sterne, J. A., Hernán, M. A., Reeves, B. C., Savović, J., Berkman, N. D., Viswanathan, M., Henry, D., Altman, D. G., Ansari, M. T., Boutron, I., Carpenter, J. R., Chan, A.-W., Churchill, R., Deeks, J. J., Hróbjartsson, A., Kirkham, J., Jüni, P., Loke, Y. K., Pigott, T. D. \& Higgins, J. P. (2016). ROBINS-I: A tool for assessing risk of bias in non-randomised studies of interventions. BMJ, vol. 355, i4919. https://doi.org/10.1136/bmj.i4919 
Tsikinas, S. \& Xinogalos, S. (2020). Towards a serious games design framework for people with intellectual disability or autism spectrum disorder. Education and Information Technologies, vol. 25, núm. 4, pp. 3405-3423. https://doi. org/10.1007/s10639-020-10124-4

Xu, Y., Lau, Y., Cheng, L. J. \& Lau, S. T. (2021). Learning experiences of game-based educational intervention in nursing students: A systematic mixed-studies review. Nurse Education Today, vol. 107, 105139. https://doi.org/10.1016/j. nedt.2021.105139

Zainuddin, Z., Chu, S. K. W., Shujahat, M. \& Perera, C. J. (2020). The impact of gamification on learning and instruction: A systematic review of empirical evidence. Educational Research Review, vol. 30, 100326. https://doi.org/10.1016/j. edurev.2020.100326 